\title{
Predictors Associated With Post-Traumatic Hydrocephalus in Patients With Head Injury Undergoing Unilateral Decompressive Craniectomy
}

\author{
Qianxin $\mathrm{Hu}^{\dagger}$, Guangfu $\mathrm{Di}^{\dagger}$, Xuefei Shao, Wei Zhou and Xiaochun Jiang* \\ Department of Neurosurgery, Yijishan Hospital, Wannan Medical College, Wuhu, China
}

OPEN ACCESS

Edited by:

Marco Sarà,

San Raffaele Cassino, Italy

Reviewed by:

Jafri Malin Abdullah,

University of Science, Malaysia

Rita Formisano,

Fondazione Santa Lucia (IRCCS), Italy

Eric Peter Thelin,

University of Cambridge,

United Kingdom

*Correspondence:

Xiaochun Jiang

jiangxiaochun2001@hotmail.com

tThese authors have contributed equally to this work.

Specialty section:

This article was submitted to Neurotrauma,

a section of the journal

Frontiers in Neurology

Received: 04 February 2018

Accepted: 26 April 2018

Published: 14 May 2018

Citation:

Hu Q, Di G, Shao X, Zhou W and Jiang $X$ (2018) Predictors Associated With Post-Traumatic Hydrocephalus in Patients With Head Injury Undergoing Unilateral Decompressive Craniectomy. Front. Neurol. 9:337. doi: 10.3389/fneur.2018.00337
Objective: Post-traumatic hydrocephalus $(\mathrm{PTH})$ makes recovery from head trauma after decompression more complicated and is associated with high risks of clinical deterioration and poor outcomes. The aim of this study was to verify the predictors associated with the development of PTH in patients with head injury undergoing unilateral decompressive craniectomy (DC).

Methods: Among traumatic brain injury (TBI) patients who underwent unilateral DC between January 2013 and December 2016, the clinical medical records, radiological information, and changes of patients' conditions in the 3-month after injury were reviewed retrospectively.

Results: 183 TBI patients after unilateral DC were analyzed, and 50 (27.32\%) of them suffered PTH based on head CT scans. Univariate and multivariable analyses revealed that older age $(p=0.002)$, the Glasgow Coma Scale (GCS) score at admission $(p<0.001)$, intraventricular hemorrhage (IVH; $p=0.008$ ), post-traumatic cerebral infarction ( $\mathrm{PCl}$; $p=0.007)$, and postoperative meningitis $(p=0.016)$ were independent predictors for the hydrocephalus after DC. Receiver operating characteristic curves were created and the area under the curve (AUC) were calculated to further assess the accuracy of the variables for predicting PTH. The AUC was 0.836 for the combined all five independent factors (95\% confidence interval: 0.775-0.887).

Conclusion: TBI patients who undergo unilateral DC with advanced age, lower GCS score at admission, coexisting $\mathrm{IVH}, \mathrm{PCl}$, and/or postoperative meningitis should be closely monitored at follow-up assessments for earlier prediction of PTH.

Keywords: post-traumatic hydrocephalus, decompressive craniectomy, traumatic brain injury, risk factor, unilateral

\section{INTRODUCTION}

Previous studies have proved decompressive craniectomy (DC) was an independent risk factor of post-traumatic hydrocephalus (PTH) after a traumatic brain injury (TBI) (1-3). PTH occurs in $11.9-36.0 \%$ of patients who undergo DC, which is a concern because PTH can lead to unfavorable outcomes (4-6). PTH is typically characterized by progressive accumulation of cerebrospinal fluid (CSF) and ventriculomegaly secondary to disorders involving CSF circulation and malabsorption. 
PTH can disrupt brain function or metabolism, delay the clinical improvement, and aggravate TBI outcomes if not be detected and effectively treated in time (7). Therefore, the early diagnosis and treatment of PTH can prevent further neurological complications in patients who are recovering from TBI. However, the findings of studies that have investigated the risk factors associated with PTH after DC are heterogenous due to different evaluation methods and enrollment criteria used.

Thus, the present study performed a retrospective review of patients undergoing unilateral DC to determine the incidence of PTH during hospitalization in the 3 months after TBI. In addition, the prognostic values of the clinical risk factors related to $\mathrm{PTH}$ were evaluated, and a predictive receiver operating characteristic (ROC) model was developed by combining the independent predictors associated with $\mathrm{PTH}$.

\section{PATIENTS AND METHODS}

\section{Patient Characteristics}

This retrospective study was approved by the Institutional Ethical Board of the Yijisha Hospital of Wannan Medical College. The institutional database was searched to identify consecutive TBI patients who underwent unilateral DC and were treated between January 2013 and December 2016. To avoid confounding variables, the following exclusion criteria were applied: patients who had (1) a past neurological history, (2) dilated ventricles or hydrocephalus present on the initial computed tomography (CT) scans or the first CTs were scanned longer than $6 \mathrm{~h}$ after injury, (3) undergone a bilateral or bifrontal craniectomy, (4) lost to follow-up, and (5) multiple injuries (abbreviated injury score $\geq 3$ ). Electronic medical records, admission, and follow-up head CT scans were reviewed by two authors. Patients with different diagnosis were also excluded.

\section{Measured Variables}

The following factors were assessed prior to DC and included in the analyses: Glasgow Coma Scale (GCS) scores at admission, hypoxemia, pupil reactivity, status of basal cistern, extent of midline shift, and the presence of traumatic subarachnoid hemorrhage (tSAH), intraventricular hemorrhage (IVH), subdural hemorrhage (SDH), epidural hemorrhage (EDH), and contusion-associated hemorrhage. Hypoxemia was defined as (1) when hemoglobin oxygen saturation was less than $90 \%$ or (2) when the partial pressure of oxygen in blood was less than $60 \mathrm{~mm} \mathrm{Hg}$ at admission. Pupil reactivity was divided into no reactive, one reactive (include "sluggish"), and both reactive. Midline shift was measured in millimeters as a continuous variable. Basal cistern compression was defined as basal cistern narrowing or disappearance, it was dichotomized as "yes" (compressed) and "no" (absent). Potential risk factors after DC were also analyzed and included craniectomy size, decompression time after injury, reoperation, post-traumatic cerebral infarction (PCI), postoperative meningitis, craniectomy margin from midline, various types of hygromas, and Glasgow Outcome Scale Extended (GOSE) at 3 months after TBI. Diagnosis of IVH can be confirmed by the presence of blood inside the ventricles with Graeb scale $>1$ on
CT (8). PCI was defined as low-attenuation lesions in well-defined arterial vascular distribution on any brain CT scan within 2 weeks after the accident, and a diagnosis of cerebral infarction was revised if follow-up studies indicated the findings were actually related to evolving contusions, artifacts, or were inconsistently visualized (9). The definition of postoperative meningitis must meet at least one of the followings: either (1) patient has organisms cultured from CSF or (2) patient has the following signs or symptoms with no other recognized cause: fever $\left(>38^{\circ} \mathrm{C}\right)$, meningeal signs and at least one of the followings: (a) increased white cell count, elevated protein, and/or decreased glucose level in CSF. (b) organisms seen on Gram's stain of CSF (10). Craniectomy size was calculated by skull X-ray for locating the head CT that was taken postoperatively, as described previously $(6,11):$ craniectomy size $=$ (largest transverse diameter $\times$ vertical diameter perpendicular to largest transverse diameter) $\times \pi / 4$. The craniectomy margin from the midline was defined as the mean of the maximum and minimum distances from the midline to the medial border of the bone flap (11). The GOSE was employed to evaluate patients at the end of 3-month follow-up.

\section{Patients' Treatment}

After admission, patients were initially scanned by CT following serial neurological examinations. When hypoxemia occurs, intubation and mechanical ventilation may be needed. Intracranial traumatic lesions associated with a midline shift $>5 \mathrm{~mm}$, volume hematomas $\geq 30 \mathrm{ml}$, basal cistern compression, abnormal pupillary reaction, or neurological deteriorations were treated with osmotic therapy or DC. Operative records were reviewed to determine surgical indications and details. There were two clinical scenarios in which DC for TBI was performed. The first scenario involved patients who had DC as part of an operation to treat a hematoma or edema or resective surgery for diffuse injury immediately after admission ("primary DC"). The second was that patients had DC for refractory malignant brain swelling despite maximal medical management ("secondary DC"). Any clinical deterioration indicated the need for another CT scan, and a ventriculoperitoneal shunt might be considered in patients with PTH. All patients were evaluated and treated as prescribed by currently accepted international guidelines (12).

\section{Definition of PTH}

All patients underwent follow-up assessment that included a neurological examination and brain $\mathrm{CT}$ scan at 3 months after the injury. The presence of PTH on the serial CT images was featured using enlargement of lateral and third ventricle or as a frontal horn index $\geq 33 \%$ with "infiltration syndrome" around ventricle on CT which was not found before $(13,14)$;. The clinical features of PTH included high flap-tension and lowering/no improvement of consciousness. Once the patients were suspected of PTH, temporary CSF drainage test was conducted, if the GCS of the patients improved $\geq 2$, we defined them as PTH.

\section{Statistical Analysis}

IBM SPSS Statistics 23 was employed for analyses. T tests or MannWhitney $U$ tests were performed to analyze continuous variables, and chi-square tests were conducted to analyze categorical ones. 
Significant factors in the univariate analysis were enrolled in a logistic regression to screen independent risk factors and then used in a predictive ROC model. The area under the curve (AUC) values in the ROC analysis were used to assess the discriminatory power of the model, which was classified as follows in terms of predictive ability: good to excellent (AUC > 0.8), moderate (AUC: $0.7-0.8$ ), and low (AUC: 0.6-0.7). Comparison of ROC curves was analyzed by the MedCalc statistical software. $p$ Values $<0.05$ were considered to indicate statistical significance.

\section{RESULTS}

A total of 183 TBI patients who underwent unilateral DC between January 2013 and December 2016 were included in the final analyses. The baseline demographic, clinical, and radiological data of the patients are shown in Table 1. During the 3-month follow-up period, 50 patients $(27.32 \%)$ developed PTH. At the diagnosis of $50 \mathrm{PTH}$ patients, urinary incontinence was found in 16 ; increased spasticity in 21; and epileptic seizures in 11 . Once the patients were diagnosed as PTH, VP shunt was considered. However, only parts of patients $(22 / 50,44 \%)$ underwent the VP shunts, others did not because of fever, pneumonia, intracranial or urinary tract infection, or high treatment costs. Follow-up in shunted patients showed symptomatic improvements in 17/22 (improvements of urinary incontinence in 6 out of 7 ; reductions of limb spasticity in 8 out of 9; and reliefs of epileptic seizures in 3 out of 6). In our trauma center, cranioplasty is traditionally conducted at more than 3 months after TBI $(15,16)$, only 4 of 183 patients underwent cranioplasty within 3-month followup. The patients who developed PTH were significantly older $(p=0.01)$ and had significantly different mean GCS scores at admission and hypoxemia and pupil reactivity statuses prior to DC, compared with those who did not develop PTH. Of the coexisting hemorrhages identified on the initial head CT scan, IVH occurred in $88.2 \%$ of cases and was significantly associated with PTH $(p=0.001)$. In addition, the PTH and non-PTH groups significantly differed in terms of basal cistern status $(p<0.001)$, midline shift $(p=0.025)$, and craniectomy size (PTH group: $79.38 \pm 16.83 \mathrm{~cm}^{2}$, non-PTH group: $\left.72.77 \pm 16.41 \mathrm{~cm}^{2} ; p=0.017\right)$.

Of the variables measured after DC, the incidences of PCI and postoperative meningitis were significantly higher in the PTH group compared with the non-PTH group $(p=0.001$ and $p=0.017$, respectively). However, interhemispheric hygroma was the only factor associated with $\mathrm{PTH}$ among all types of hygromas $(p=0.017)$. The GOSE scores of the PTH patients were significantly lower at the 3-month post-injury assessment compared with the non-PTH patients $(p<0.001)$. The multivariable analysis revealed that older age $(p=0.002)$, GCS score at admission $(p<0.001)$, and the presence of IVH $(p=0.008)$, PCI $(p=0.007)$, and postoperative meningitis $(p=0.016)$ were independently associated with PTH after DC (Table 2).

To further assess the accuracy of the factors that may predict $\mathrm{PTH}, \mathrm{ROC}$ curves were created, and the AUC values were calculated (Figure 1). The AUC was 0.615 [95\% confidence interval (CI): $0.540-0.685$ ] for age (with a cut-off value of $>51$ years), 0.715 (95\% CI: 0.644-0.779) for GCS scores at admission (with a cut-off value of < 6), 0.596 (95\% CI: 0.521-0.668) for IVH, 0.601 (95\% CI: 0.526-0.673) for PCI, and 0.542 (95\% CI: 0.467-0.616) for postoperative meningitis (Table 3). Finally, the AUC for these five factors combined was 0.836 (95\% CI: 0.775-0.887), with a sensitivity of $86 \%$ and a specificity of $72 \%$. These findings indicate that the combined five factors exhibit excellent predictive power for PTH.

\section{DISCUSSION}

Post-traumatic hydrocephalus is a common and devastating complication that may occur following TBI. Although various studies have reported the incidence of PTH $(1,17)$, few have specifically analyzed TBI patients who underwent unilateral DC or focused on the risk factors for PTH in this subgroup. Thus, the present study diagnosed PTH according to changes of imaging or clinical features and identified a PTH incidence of $27.32 \%$. Moderate and severe TBI patients without DC between January 2013 and December 2016 in our center were also reviewed and only 9 out of $112(8.0 \%)$ developed a PTH at 3-month post-injury. These results showed this sub-cohort might actually be more susceptible to PTH. In our trauma center, more than 300 patients with moderate-to-severe TBI are admitted each year. PTH may be easily overlooked during rehabilitation and its consequences are extremely destructive. Thus, early prediction of hydrocephalus will be valuable for TBI evaluation after DC and for improved outcomes. In the present study, univariate and multivariable analyses were conducted to determine the risk factors associated with PTH after DC; the independent predictive factors included older age, lower GCS score at admission, and the presence of IVH, PCI, and postoperative meningitis after DC. The ROC curve for these five factors integrated provided good discrimination of PTH risk, with an AUC that was indicative of excellent predictive power.

Advanced age is an influential factor in the development of PTH in TBI patients $(1,17)$. Older patients have a wider subarachnoid space that can hold larger volumes of subarachnoid blood, which increases their risk of developing disturbances in CSF circulation (18). It is also possible that the extent of meningeal fibrosis, which impairs CSF circulation and decreases CSF absorption, is greater in elderly patients (19). In the present study, the ROC for the age determined the cut-off value of the age was 51 , which means when the age $>51$ years, the probability of PTH significantly increased. A lower GCS score at admission was also a risk factor of hydrocephalus in our study, which is in correspondence with prior findings $(1,5,17)$. Patients with lower GCS scores or severe consciousness disorders generally suffer from grave disturbances in CSF formation or absorption and increasing the risk of development of hydrocephalus.

Other potential risk factors for PTH include various types of coexisting hemorrhages evident on a CT scan $(2,20)$. However, in the present study, only IVH was significantly associated with $\mathrm{PTH}$, while $\mathrm{tSAH}, \mathrm{SDH}$, and $\mathrm{EDH}$ were not. Severe IVH after TBI promotes the formation of hydrocephalus and abnormal CSF flow through an obstructive mechanism. Small blood clots or other substances can obstruct CSF circulation at the arachnoid 
TABLE 1 | Demographic, clinical, and radiological data for 183 patients who underwent DC procedures after traumatic brain injury.

\begin{tabular}{|c|c|c|c|c|}
\hline & & & & $p$ \\
\hline Mean age in years (SD) & $53.04 \pm 14.36$ & $57.46 \pm 13.76$ & $51.38 \pm 14.27$ & 0.01 \\
\hline Sex & & & & 0.214 \\
\hline Male & 133 & 33 & 100 & \\
\hline GCS scores on admission (SD) & $7 \pm 2$ & $6 \pm 2$ & $7 \pm 2$ & $<0.001$ \\
\hline Hypoxemia & & & & 0.001 \\
\hline Pupil reactivity & & & & $<0.001$ \\
\hline None & 47 & 23 & 24 & \\
\hline One reactive & 31 & 12 & 19 & \\
\hline Both reactive & 105 & 15 & 90 & \\
\hline Type of DC & & & & 0.453 \\
\hline Primary DC & 74 & 18 & 56 & \\
\hline $\mathrm{IVH}$ & & & & $<0.001$ \\
\hline Yes & 22 & 13 & 9 & \\
\hline No & 161 & 37 & 124 & \\
\hline $\mathrm{SDH}$ & & & & 0.667 \\
\hline Yes & 143 & 38 & 105 & \\
\hline No & 40 & 12 & 28 & \\
\hline EDH & & & & 0.632 \\
\hline Yes & 41 & 10 & 31 & \\
\hline No & 142 & 40 & 102 & \\
\hline Contusion-associated hemorrhage & & & & 0.172 \\
\hline Yes & 158 & 46 & 112 & \\
\hline No & 25 & 4 & 21 & \\
\hline Status of basal cistern & & & & $<0.001$ \\
\hline Yes & 35 & 11 & 24 & \\
\hline No & 148 & 39 & 109 & \\
\hline $\mathrm{PCl}$ & & & & 0.001 \\
\hline Yes & 28 & 15 & 13 & \\
\hline No & 155 & 35 & 120 & \\
\hline Craniectomy margin from midline $(\mathrm{cm})$ & & & & 0.076 \\
\hline$\leqq 2.5$ & 83 & 28 & 55 & \\
\hline$>2.5$ & 100 & 22 & 78 & \\
\hline Subdural hygroma & & & & 0.055 \\
\hline Yes & 115 & 37 & 78 & \\
\hline No & 68 & 13 & 55 & \\
\hline Interhemispheric hygroma & & & & 0.013 \\
\hline Yes & 25 & 12 & 13 & \\
\hline No & 158 & 38 & 120 & \\
\hline Bilateral hygroma & & & & 0.678 \\
\hline Yes & 9 & 3 & 6 & \\
\hline No & 174 & 47 & 127 & \\
\hline Ipsilateral hygroma & & & & 0.277 \\
\hline Yes & 74 & 17 & 57 & \\
\hline No & 109 & 33 & 76 & \\
\hline Contralateral hygroma & & & & 0.026 \\
\hline Yes & 13 & 7 & 6 & \\
\hline No & 170 & 43 & 127 & \\
\hline
\end{tabular}


TABLE 1 | Continued

\begin{tabular}{|c|c|c|c|c|}
\hline & & \multicolumn{2}{|c|}{ PTH } & \multirow{2}{*}{$\begin{array}{c}\boldsymbol{p} \\
0.008\end{array}$} \\
\hline & & & & \\
\hline Yes & 7 & 5 & 2 & \\
\hline No & 176 & 45 & 131 & \\
\hline GOSE (SD) & $5 \pm 2$ & $3 \pm 2$ & $6 \pm 2$ & $<0.001$ \\
\hline Shunted (22) & - & $4 \pm 2$ & - & \\
\hline Non-shunted (28) & - & $2 \pm 0$ & - & \\
\hline
\end{tabular}

GCS, Glasgow Coma Scale; tSAH, traumatic subarachnoid hemorrhage; IVH, intraventricular hemorrhage; SDH, subdural hemorrhage; EDH, epidural hemorrhage;

PCl, post-traumatic cerebral infarction; GOSE, Glasgow Outcome Scale Extended; PTH, post-traumatic hydrocephalus; DC, decompressive craniectomy.

TABLE 2 | Multiple logistic regression model for factors associated with PTH.

\begin{tabular}{lccr}
\hline Factors & OR & $\mathbf{9 5 \%} \mathbf{C l}$ & $\boldsymbol{p}$ Value \\
\hline Age & 1.052 & $1.109-1.087$ & 0.002 \\
GCS scores on admission & 0.651 & $0.527-0.803$ & $<0.001$ \\
IVH & 4.091 & $1.435-11.658$ & 0.008 \\
PCl & 4.059 & $1.469-11.214$ & 0.007 \\
Postoperative meningitis & 11.876 & $1.578-89.388$ & 0.016
\end{tabular}

OR, odds ratio; Cl, confidence interval; GCS, Glasgow Coma Scale;

$\mathrm{NH}$, intraventricular hemorrhage; $\mathrm{PCl}$, post-traumatic cerebral infarction;

PTH, post-traumatic hydrocephalus.

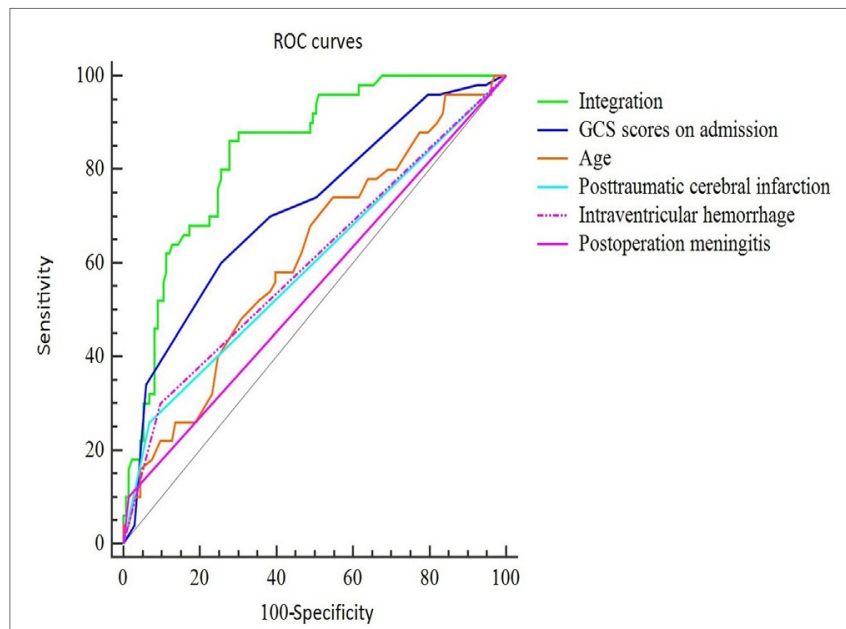

FIGURE 1 | ROC curves of the final predictive model using five main risk factors. The sensitivity and specificity of the final combined curve were 0.86 and 0.72 , respectively. Abbreviations: GCS, Glasgow Coma Scale; IVH, intraventricular hemorrhage; $\mathrm{PCl}$, post-traumatic cerebral infarction.

TABLE 3 | The AUCs under the ROC curves.

\begin{tabular}{lccc}
\hline & AUC & SE & $\mathbf{9 5 \%} \mathbf{~ C l}$ \\
\hline Combined & 0.836 & 0.0310 & $0.775-0.887$ \\
Age & 0.615 & 0.0468 & $0.540-0.685$ \\
GCS scores on admission & 0.715 & 0.0432 & $0.644-0.779$ \\
IVH & 0.596 & 0.0332 & $0.521-0.668$ \\
PCl & 0.601 & 0.0352 & $0.526-0.673$ \\
Postoperative meningitis & 0.542 & 0.0221 & $0.467-0.616$
\end{tabular}

AUC, area under the curve; ROC, receiver operating characteristic; GCS, Glasgow

Coma Scale; $\mathrm{VH}$, intraventricular hemorrhage; $\mathrm{PCl}$, post-traumatic cerebral infarction;

$\mathrm{Cl}$, confidence interval. granulation site or block the fourth ventricle or sacra fistula directly, which would cause decreased CSF absorption, increased CSF accumulation, and ultimate formation of PTH (21).

The craniectomy size was bigger in the PTH group, it has been suggested that craniectomy may play a role in the flattening of the normal dicrotic intracranial waveform due to the transmission of the pressure pulse out through the open cranium, which leads to decreased CSF outflow to the arachnoid granulations $(11,13)$. Thus, a bigger craniectomy size may be a risk factor for the development of hydrocephalus, via an impairment of hydrodynamic CSF circulation. In the PTH patients, another important reason of using a larger craniectomy may be more severe injury or lower admission GCS scores. The real mechanism of its effect on PTH remains unclear. According to our results, the craniectomy size did not affect the development of PTH independently, but we should not neglect its role in future study.

In the present study, the incidence of PCI was significantly higher in the PTH group than in the non-PTH group and was an independent risk factor for hydrocephalus. PCI is a severe and common complication in patients with head trauma, and blunt cerebral vascular injuries, hypotension, high ICP, low GCS scores, brain herniation, and DC may be risk factors for PCI in patients with moderate or severe TBI $(9,22)$. Cerebral infarctions after TBI may lead to reduced regional cerebral perfusion as well as microangiopathy, hypoxia, necrosis, and even neurological deficits. In addition, in communicating hydrocephalus, disorders involving the CSF and cerebrovascular circulation are associated with one another $(23,24)$, but the causal link between these factors remains unknown. Peña and colleagues $(23,25)$ proposed that the ventricular dilatation associated with communicating hydrocephalus results from a reversal of interstitial fluid flow into the parenchyma and reduced tissue elasticity. This may explain the relationship between PCI and PTH.

Postoperative meningitis or infections may be among the most common causes of hydrocephalus (26), which is supported by the present results. Chen et al. (1) found no evidence of a correlation between intracranial infections and PTH, but this may have been due to the use of continuous lumbar CSF drainage in their patients. A prior study (2), which demonstrated that the continuous lumbar subarachnoid drainage significantly reduces the hydrocephalus after TBI, confirmed this deduction.

Finally, an ROC model was created in the present study to further assess the accuracy of these factors for predicting PTH. Based on the AUC values, only the combined all five factors provided a good discrimination of PTH risk, and the AUC was 
indicative of excellent predictive power. Independently, GCS score at admission had a moderate predictive accuracy, whereas the other factors (age and the presence of IVH, PCI, and postoperative meningitis) had low predictive values.

Traumatic brain injury is still a main reason of morbidity and disability among trauma groups, which is also responsible for a notable ratio of all traumatic deaths. The disability and loss of human potential and long-term impairment resulted from TBI has led to enormous influence to the society and family as well as costs society a significant amount of money each year. In our trauma center, once there is a surgical indication, immediate DC is performed to save the patient's life in despite of lack of money. If the injury was caused by traffic accident, the person who caused the accident will pay the victim. TBI caused by other reason may be paid by their families, or their social and commercial insurance agents. The high medical costs and insufficient insurance have brought the financial burden to the poor families. Unfortunately, some of them may postpone or opt out from the beneficial treatments. Although early prediction and diagnosis of PTH may help to decrease the hospital days and expenses, our government and community may still need to take effective measures, such as increasing the use of safety belts, and increasing personal protection for dangerous work to prevent and reduce the incidence of TBI.

There were several limitations to this study. First, due to only parts of PTH patients underwent the VP shunts, relatively few cases of VP-shunt-conducted hydrocephalus limited the power to identify risk factors for the development of VP-shuntconducted PTH. Second, this was a retrospective study based on neurological examinations and radiological findings that had a limitation of evaluating whether patients might benefit from

\section{REFERENCES}

1. Chen H, Yuan F, Chen SW, Guo Y, Wang G, Deng ZF, et al. Predicting posttraumatic hydrocephalus: derivation and validation of a risk scoring system based on clinical characteristics. Metab Brain Dis (2017) 32(5):1427-35. doi:10.1007/s11011-017-0008-2

2. Jiao QF, Liu Z, Li S, Zhou LX, Li SZ, Tian W, et al. Influencing factors for posttraumatic hydrocephalus in patients suffering from severe traumatic brain injuries. Chin J Traumatol (2007) 10(3):159-62.

3. Mazzini L, Campini R, Angelino E, Rognone F, Pastore I, Oliveri G. Posttraumatic hydrocephalus: a clinical, neuroradiologic, and neuropsychologic assessment of long-term outcome. Arch Phys Med Rehabil (2003) 84:1637-41. doi:10.1053/S0003-9993(03)00314-9

4. De Bonis P, Sturiale CL, Anile C, Gaudino S, Mangiola A, Martucci M, et al. Decompressive craniectomy, interhemispheric hygroma and hydrocephalus: a timeline of events. Clin Neurol Neurosurg (2013) 115(8):1308-12. doi:10.1016/j.clineuro.2012.12.011

5. Honeybul S, Ho KM. Incidence and risk factors for post-traumatic hydrocephalus following decompressive craniectomy for intractable intracranial hypertension and evacuation of mass lesions. JNeurotrauma (2012) 29(10):1872-8. doi:10.1089/neu.2012.2356

6. Ki HJ, Lee HJ, Lee HJ, Yi JS, Yang JH, Lee IW. The risk factors for hydrocephalus and subdural hygroma after decompressive craniectomy in head injured patients. J Korean Neurosurg Soc (2015) 58(3):254-61. doi:10.3340/ jkns.2015.58.3.254

7. Licata C, Cristofori L, Gambin R, Vivenza C, Turazzi S. Post-traumatic hydrocephalus. J Neurosurg Sci (2001) 45(3):141-9.

8. Yang XT, Feng DF, Zhao L, Sun ZL, Zhao G. Application of the Ommaya reservoir in managing ventricular hemorrhage. World Neurosurg (2016) 89:93-100. doi:10.1016/j.wneu.2015.12.040 shunt operation for CSF diversion. Therefore, future research should focus on recruiting more cases, and should implement long-term follow-up and a more thorough review of patients in a prospective manner.

\section{CONCLUSION}

The present study demonstrated that the risk of PTH can be independently predicted by older age, lower GCS score at admission, PCI, IVH, and intracranial infection after DC. However, a combination of these five factors provided much stronger predictive power. In the future, comprehensive analyses should be conducted in high-risk TBI patients undergoing unilateral DC to better understand the relationships among these factors.

\section{ETHICS STATEMENT}

This retrospective study was approved by the Institutional Ethical Board of the Yijisha Hospital of Wannan Medical College.

\section{AUTHOR CONTRIBUTIONS}

$\mathrm{QH}$ and XJ designed the experiments; GD and XS performed data analysis; WZ provided scientific expertise; $\mathrm{QH}$ wrote the manuscript. Because QH and GD contributed equally to this work, they are considered as co-first authors.

\section{FUNDING}

This study was supported by Anhui University Natural Science Fund Project (KJ2015ZD40).

9. Tian HL, Geng Z, Cui YH, Hu J, Xu T, Cao HL, et al. Risk factors for posttraumatic cerebral infarction in patients with moderate or severe head trauma. Neurosurg Rev (2008) 31(4):431-6; discussion 436-7. doi:10.1007/ s10143-008-0153-5

10. Chen C, Zhang B, Yu S, Sun F, Ruan Q, Zhang W, et al. The incidence and risk factors of meningitis after major craniotomy in China: a retrospective cohort study. PLoS One (2014) 9:e101961. doi:10.1371/journal.pone.0101961

11. Kim H, Lee HS, Ahn SY, Park SC, Huh W. Factors associated postoperative hydrocephalus in patients with traumatic acute subdural hemorrhage. J Korean Neurosurg Soc (2017) 60(6):730-7. doi:10.3340/jkns.2017.0210

12. Carney NA, Ghajar J. Guidelines for the management of severe traumatic brain injury. Introduction. J Neurotrauma (2007) 24(Suppl 1):S1-2. doi:10.1089/ neu.2007.9997

13. Foroglou G, Zander E. [Post-traumatic hydrocephalus and measurement of cerebrospinal fluid pressure]. Acta Radiol Diagn (Stockh) (1972) 13(0):524-30. doi:10.1177/02841851720130P206

14. Fotakopoulos G, Tsianaka E, Siasios G, Vagkopoulos K, Fountas K. Posttraumatic hydrocephalus after decompressive craniectomy in 126 patients with severe traumatic brain injury. J Neurol Surg A Cent Eur Neurosurg (2016) 77(2):88-92. doi:10.1055/s-0035-1558411

15. Schuss P, Vatter H, Marquardt G, Imöhl L, Ulrich CT, Seifert V, et al. Cranioplasty after decompressive craniectomy: the effect of timing on postoperative complications. J Neurotrauma (2012) 29:1090-5. doi:10.1089/ neu.2011.2176

16. Quah BL, Low HL, Wilson MH, Bimpis A, Nga VDW, Lwin S, et al. Is there an optimal time for performing cranioplasties? Results from a prospective multinational study. World Neurosurg (2016) 94:13-7. doi:10.1016/j. wneu.2016.06.081

17. Kammersgaard LP, Linnemann M, Tibaek M. Hydrocephalus following severe traumatic brain injury in adults. Incidence, timing, and clinical predictors 
during rehabilitation. NeuroRehabilitation (2013) 33(3):473-80. doi:10.3233/ NRE-130980

18. Yoshioka H, Inagawa T, Tokuda Y, Inokuchi F. Chronic hydrocephalus in elderly patients following subarachnoid hemorrhage. Surg Neurol (2000) 53(2):119-24; discussion 124-5. doi:10.1016/S0090-3019(99)00185-8

19. Czosnyka M, Czosnyka ZH, Whitfield PC, Donovan T, Pickard JD. Age dependence of cerebrospinal pressure-volume compensation in patients with hydrocephalus. J Neurosurg (2001) 94(3):482-6. doi:10.3171/jns.2001.94.3.0482

20. Vedantam A, Yamal JM, Hwang H, Robertson CS, Gopinath SP. Factors associated with shunt-dependent hydrocephalus after decompressive craniectomy for traumaticbrain injury.J Neurosurg(2017) 128(5):1547-52. doi:10.3171/2017.1. JNS162721

21. Symss NP, Oi S. Theories of cerebrospinal fluid dynamics and hydrocephalus: historical trend. JNeurosurg Pediatr (2013) 11(2):170-7. doi:10.3171/2012.3.PEDS0934

22. Chen H, Xue LX, Guo Y, Chen SW, Wang G, Cao HL, et al. The influence of hemocoagulation disorders on the development of posttraumatic cerebral infarction and outcome in patients with moderate or severe head trauma. Biomed Res Int (2013) 2013:685174. doi:10.1155/2013/685174

23. Haubrich C, Czosnyka M, Diehl R, Smielewski P, Czosnyka Z. Ventricular volume load reveals the mechanoelastic impact of communicating hydrocephalus on dynamic cerebral autoregulation. PLoS One (2016) 11(7):e0158506. doi:10.1371/journal.pone.0158506
24. Momjian S, Owler BK, Czosnyka Z, Czosnyka M, Pena A, Pickard JD. Pattern of white matter regional cerebral blood flow and autoregulation in normal pressure hydrocephalus. Brain (2004) 127(Pt 5):965-72. doi:10.1093/brain/ awh131

25. Peña A, Harris NG, Bolton MD, Czosnyka M, Pickard JD. Communicating hydrocephalus: the biomechanics of progressive ventricular enlargement revisited. Acta Neurochir Suppl (2002) 81:59-63.

26. De Bonis P, Pompucci A, Mangiola A, Rigante L, Anile C. Post-traumatic hydrocephalus after decompressive craniectomy: an underestimated risk factor. J Neurotrauma (2010) 27(11):1965-70. doi:10.1089/neu.2010.1425

Conflict of Interest Statement: The authors declare that the research was conducted in the absence of any commercial or financial relationships that could be construed as a potential conflict of interest.

Copyright $\odot 2018 \mathrm{Hu}, \mathrm{Di}$, Shao, Zhou and Jiang. This is an open-access article distributed under the terms of the Creative Commons Attribution License (CC BY). The use, distribution or reproduction in other forums is permitted, provided the original author(s) and the copyright owner are credited and that the original publication in this journal is cited, in accordance with accepted academic practice. No use, distribution or reproduction is permitted which does not comply with these terms. 